Ann Allergy Asthma Immunol. 2018 July ; 121(1): 128-130. doi:10.1016/j.anai.2018.03.027.

\title{
Mast Cell Disorders: Protean Manifestations and Treatment Responses
}

\author{
Meng Chen, MD ${ }^{1}$, Alexander Kim, MD¹, Bruce Zuraw, MD ${ }^{1,2}$, Taylor A. Doherty, $\mathbf{M D}^{1}$, and \\ Sandra Christiansen, MD ${ }^{1}$ \\ ${ }^{1}$ Department of Medicine, University of California San Diego \\ ${ }^{2}$ Veterans Affairs, San Diego, CA
}

\section{Keywords}

Mast cell; Mast cell activation syndrome; Systemic mastocytosis; Omalizumab

\begin{abstract}
Mast cell disorders represent an emerging disease category notable for the diverse spectrum of presentations. Patients are clinically characterized by symptoms reflective of mast cell mediator release, which often includes episodes of urticaria with or without angioedema and flushing as well as episodes of recurrent potentially life-threatening anaphylaxis. Multiple systems can be targeted including cardiovascular, gastrointestinal (GI), psychiatric, respiratory and musculoskeletal. In addition, patients may have vague nonspecific complaints, such as abdominal discomfort or "brain fog". Triggers are often unknown and episodes follow an unpredictable pattern. The diagnosis can be elusive due to the variable clinical history and laboratory values that fluctuate depending on time of collection in relationship to a symptomatic episode.
\end{abstract}

To heighten awareness of this disease and inform clinicians on therapeutic options, we describe a cohort of 7 patients with systemic mast cell disorders. For a diagnosis of mast cell activation syndrome (MCAS) ${ }^{1}$, patients require 3 out of 3 diagnostic criteria: (1) intermittent symptoms of mast cell activation involving at least 2 organ systems, (2) improvement with pharmacologic therapy targeting mast cell mediators, (3) laboratory evidence of increased mast cell mediators (commercially available and validated markers of mast cell activation include serum tryptase, urinary N-methylhistamine, prostaglandin D2 and its metabolite 11$\beta$-Prostaglandin F2, and leukotriene E4). Systemic mastocytosis (SM) ${ }^{2}$ patients require 1 major criterion (atypical mast cell clusters in bone marrow) and 1 minor criterion or at least 3 minor criteria: (1) tryptase $>20 \mathrm{ng} / \mathrm{mL}$, (2) atypical mast cell morphology, (3) aberrant expression of C2 and CD25 on mast cells, or (4) presence of the D816V c-Kit mutation.

\footnotetext{
Corresponding author: Meng Chen, 8899 University City Lane, Suite 230, San Diego, CA 92122, Mec079@ucsd.edu. Trial Registration: Not applicable

Conflicts of interest: None
}

Publisher's Disclaimer: This is a PDF file of an unedited manuscript that has been accepted for publication. As a service to our customers we are providing this early version of the manuscript. The manuscript will undergo copyediting, typesetting, and review of the resulting proof before it is published in its final citable form. Please note that during the production process errors may be discovered which could affect the content, and all legal disclaimers that apply to the journal pertain. 
Based on these criteria patients one through five were categorized with MCAS and patients six and seven were diagnosed with indolent SM (table I)

Patients one, two and four had markedly different presumed secondary activation profiles. All had evidence of either IgE or non-IgE secondary MCAS but also exhibited abnormal serum and/or urinary markers. The presence of secondary triggers does not always equate to secondary mast cell activation. Both systemic venom responses and hypotensive anaphylaxis, for example, may be due to an underlying primary mast cell disorder. Patient one presented with recurrent anaphylaxis from various foods including one episode in which intubation was narrowly avoided. Concerning in this case were the recurrent severe anaphylaxis episodes and persistently abnormal baseline tryptase elevations. As such, patient one may warrant close surveillance despite his presumed secondary MCAS classification for evolving SM. Patient two exhibited mast cell activation due to an opioid medication.

Emerging evidence has described non-IgE-mediated mast cell activation of opioids involving the MRGPRX2 receptor ${ }^{2}$. Patient three manifested cutaneous, respiratory and GI symptoms with elevated tryptase and urinary markers. Patient four was diagnosed with Kounis syndrome in which ST-elevation myocardial infarction immediately followed pork consumption. Pork-specific IgE was positive and galactose-alpha-1,3-galactose IgE testing was negative. Kounis syndrome is a rare disorder characterized by an acute coronary syndrome triggered by the release of inflammatory mediators following an allergic insult, and has been described with underlying MCAS $^{3}$. Patient five with recurrent idiopathic anaphylaxis (IA) had a normal baseline tryptase with meaningful (>20\% above baseline tryptase plus $2 \mathrm{ng} / \mathrm{mL}$ ) symptomatic elevation along with increased urinary markers thus meeting criteria for the IA subset of $\mathrm{MCAS}^{1}$.

Both SM patients presented with abdominal pain, diarrhea, urticaria and rash (table I). Patient six experienced anaphylaxis, while patient seven reported severe, debilitating bone pain. Bone marrow biopsy confirmed mast cell infiltrates in both patients. Patient six achieved symptomatic improvement with antihistamines and cromolyn. Patient seven failed maximal medical therapy with antihistamines, montelukast, cromolyn and hydroxyurea ${ }^{4}$. $\mathrm{He}$ was subsequently started on omalizumab with dramatic improvement.

The diagnosis of mast cell disorders can be challenging given the heterogeneous clinicopathologic spectrum and the short half-lives of the available biomarkers. Tryptase is optimally collected within 4 hours after symptom onset. This time point is often overlooked by physicians tasked with emergently stabilizing a patient. In our cohort, patients 1-3 had elevated tryptase levels. Patients 2 and 3 had elevated urine markers as well. Patient four's tryptase level was not collected until 24 hours after symptom onset and was within normal limits at that timepoint; the urinary marker, however, was persistently elevated. A weakness of our report is the failure to obtain 2 or more collections of urinary mediators during a symptomatic episode or elevation of tryptase over baseline for patients 1 and 4 to meet all MCAS criteria ${ }^{1}$. Patient 2 did have a meaningful tryptase elevation; however, the timing was not delineated in the record. These omissions reflect the 'real world' challenges with patients presenting at multiple sites for emergent care. 
There is limited information available to assist clinicians in choosing effective therapies for mast cell disorders. Given the rarity of these conditions, case reports are of unique value for practitioners to guide treatment decisions and monitor outcomes. Our therapeutic approach mirrors the available literature incorporating escalating doses of antihistamines, addition of a cysteinyl leukotriene receptor blocker or 5-lipoxygenase inhibitor and/or mast cell stabilizer, particularly for GI symptoms ${ }^{5}$. Aspirin therapy can be considered, especially in patients with coexisting coronary artery disease, given evidence that prostaglandins including prostaglandin D2 may play a role in mast cell activation ${ }^{6}$. As exemplified by our cohort, response to therapy is quite variable. While one patient was adequately controlled on antihistamines as needed, the majority of patients required combination therapies. Two patients failed maximal medical therapy but achieved symptom relief with omalizumab $300 \mathrm{mg}$ every 4 weeks.

Previous case reports have suggested that omalizumab can provide symptomatic relief in patients with SM and monoclonal MCAS ${ }^{7-9}$. Omalizumab has been reported to be effective in a pediatric patient with $\mathrm{MCAS}^{10}$. Our patient 5 represents the first adult (non-clonal) MCAS patient successfully treated with omalizumab to the best of our knowledge. The mechanism by which omalizumab mediates symptom relief in mast cell disorders has not been well delineated.

Our cases highlight the variable presentation, diagnostic challenges and response to treatment of mast cell disorders. Importantly, two of these cases demonstrate that symptoms refractory to traditional medical management may be alleviated with omalizumab.

\section{Acknowledgments}

Funding Source: This work was supported by NIH grant T32 AI 007469

\section{References}

1. Akin C. Mast cell activation syndromes. Journal of Allergy and Clinical Immunology. 2017; 140:349-55. [PubMed: 28780942]

2. McNeil BD, Pundir P, Meeker S, et al. Identification of a mast-cell-specific receptor crucial for pseudo-allergic drug reactions. Nature. 2015; 519:237-41. [PubMed: 25517090]

3. Abdelghany M, Subedi R, Shah S, Kozman H. Kounis syndrome: A review article on epidemiology, diagnostic findings, management and complications of allergic acute coronary syndrome. International Journal of Cardiology. 2017; 232:1-4. [PubMed: 28153536]

4. Pardanani A. Systemic mastocytosis in adults: 2017 update on diagnosis, risk stratification and management. Am J Hematol. 2016; 91:1146-59. [PubMed: 27762455]

5. Theoharides TC, Valent P, Akin C. Mast Cells, Mastocytosis, and Related Disorders. N Engl J Med. 2015; 373:163-72. [PubMed: 26154789]

6. Ravi A, Butterfield J, Weiler CR. Mast cell activation syndrome: improved identification by combined determinations of serum tryptase and 24-hour urine $11 \beta$-prostaglandin $2 a$. J Allergy Clin Immunol Pract. 2014; 2:775-8. [PubMed: 25439370]

7. Jagdis A, Vadas P. Omalizumab effectively prevents recurrent refractory anaphylaxis in a patient with monoclonal mast cell activation syndrome. Annals of Allergy, Asthma \& Immunology. 2014; 113:115-6.

8. Lieberoth S, Thomsen SF. Cutaneous and Gastrointestinal Symptoms in Two Patients with Systemic Mastocytosis Successfully Treated with Omalizumab. Case Reports in Medicine. 2015; 2015:1-3. 
9. Kibsgaard L, Skjold T, Deleuran M, Vestergaard C. Omalizumab Induced Remission of Idiopathic Anaphylaxis in a Patient Suffering from Indolent Systemic Mastocytosis. Acta Derm Venerol. 2014; 94:363-4. [PubMed: 24162824]

10. Bell MC, Jackson DJ. Prevention of anaphylaxis related to mast cell activation syndrome with omalizumab. Annals of Allergy, Asthma \& Immunology. 2012; 108:383-4. 


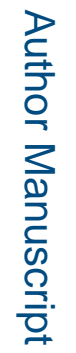

\begin{tabular}{|c|c|c|c|c|c|c|}
\hline 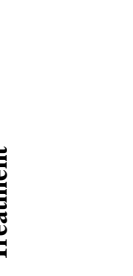 & 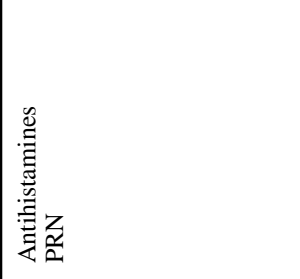 & 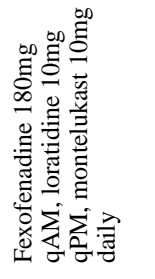 & 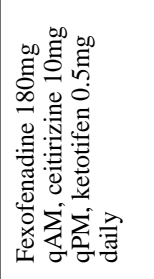 & 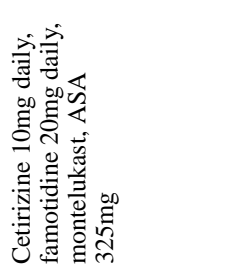 & 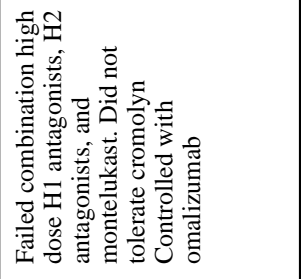 & 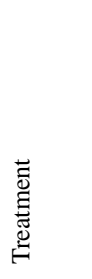 \\
\hline te & $\bar{z}$ & 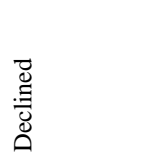 & $\mid \begin{array}{l}\tilde{x} \\
\ddot{z}\end{array}$ & 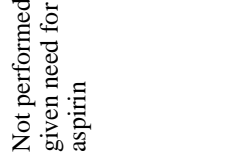 & $\bar{z}$ & \multirow[b]{5}{*}{ 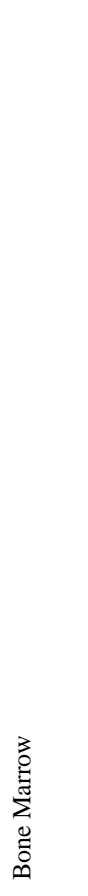 } \\
\hline 论藏 & $\bar{z}$ & $\bar{z}$ & 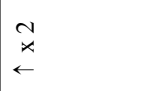 & $\bar{z}$ & $\bar{z}$ & \\
\hline 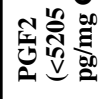 & $\bar{z}$ & $\leftarrow$ & $\begin{array}{l}\tilde{x} \\
\dot{z}\end{array}$ & $\bar{z}$ & $\leftarrow$ & \\
\hline 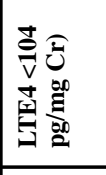 & $\bar{z}$ & 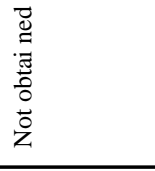 & $\begin{array}{l}\tilde{x} \\
\ddot{z} \\
\end{array}$ & $\bar{z}$ & $\leftarrow$ & \\
\hline 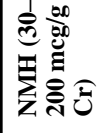 & $\bar{z}$ & $\bar{z}$ & $\begin{array}{l}\tilde{N} \\
\stackrel{x}{z}\end{array}$ & $\leftarrow$ & $\bar{z}$ & \\
\hline 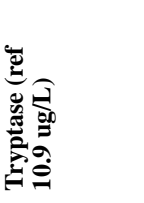 & 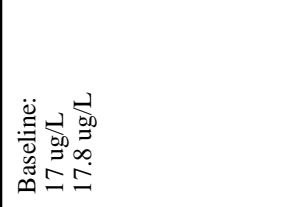 & 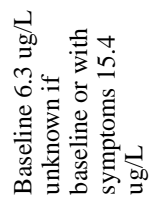 & 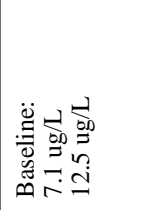 & 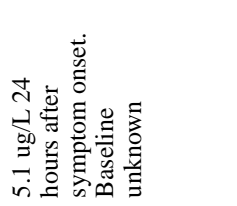 & 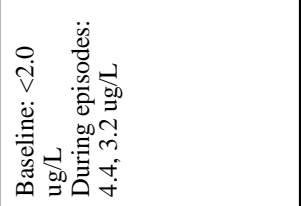 & 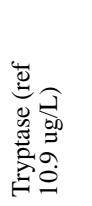 \\
\hline है & 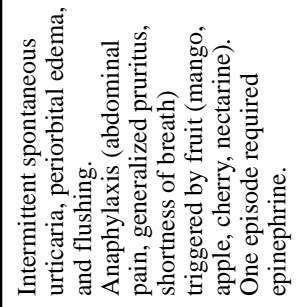 & 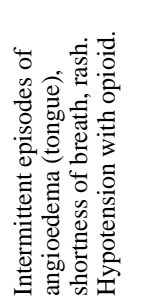 & 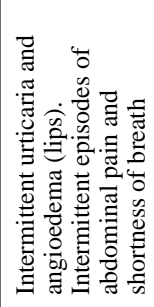 & 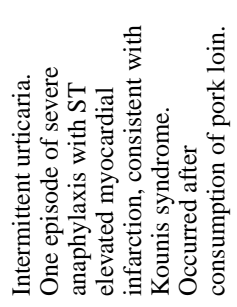 & 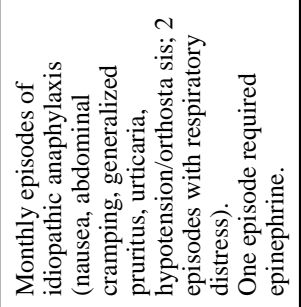 & 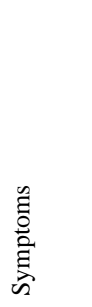 \\
\hline 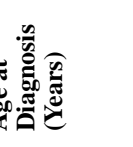 & $\stackrel{\infty}{q}$ & in & f & f & $\stackrel{\infty}{\sim}$ & 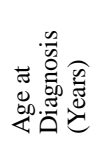 \\
\hline & $\Sigma$ & 山 & $\Sigma$ & $\Sigma$ & 幽 & 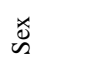 \\
\hline & - & $N$ & $m$ & $\sigma$ & in & 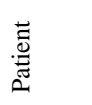 \\
\hline
\end{tabular}

Ann Allergy Asthma Immunol. Author manuscript; available in PMC 2019 July 01. 


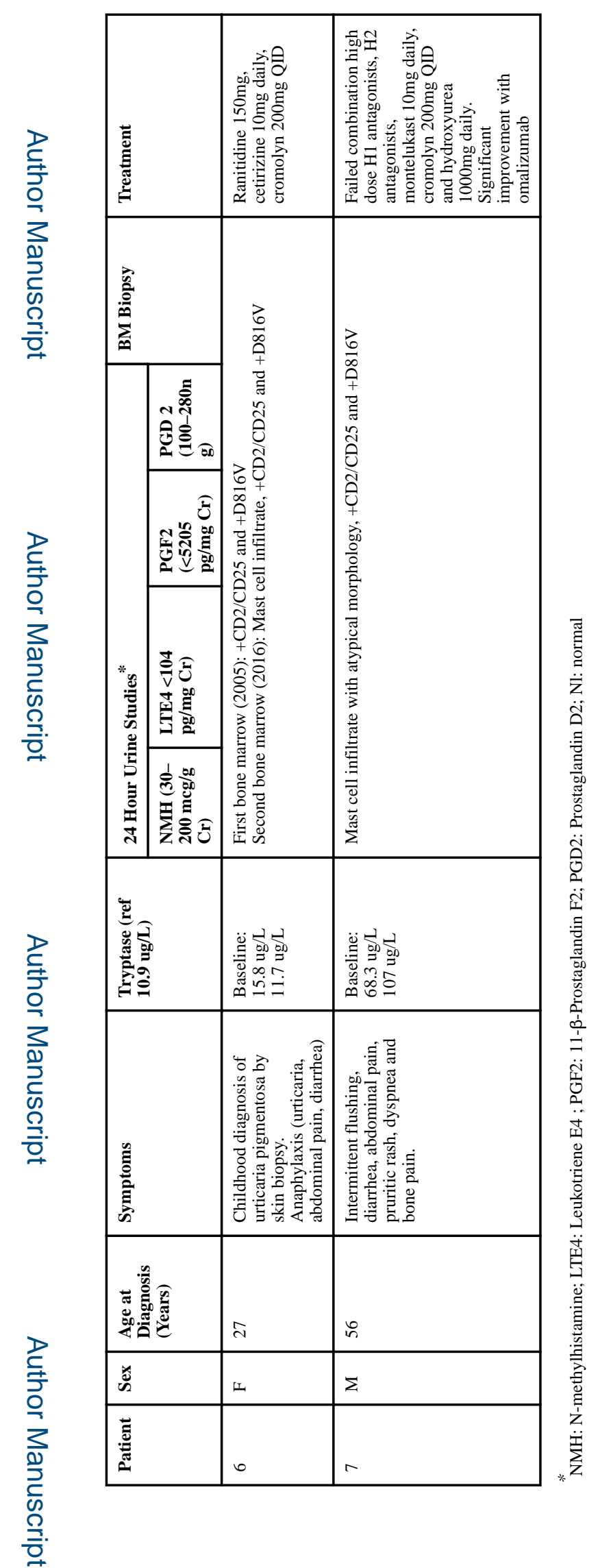

Ann Allergy Asthma Immunol. Author manuscript; available in PMC 2019 July 01. 